number of flights per day (adjusted OR 2.0, 95\% CI 1.2-3.3 for $3+$ versus 1 flights/day, $\mathrm{p}$ trend $=0.007$ ) and fewer time zones crossed per flight (adjusted OR 2.0, 95\% CI 1.0-3.7 for 0 vs. 2 + time zones/flight, $\mathrm{p}$ trend $=0.04$ ) were associated with a higher rate of endometriosis.

Conclusions The rate of endometriosis increased with number of flights and decreased with time zones crossed, which might be surrogates for exposures specific to flying a series of short flights during the workday.

\section{NONMALIGNANT DISEASE MORTALITY AMONG STYRENE, FIBREGLASS, AND WOOD DUST EXPOSED WORKERS IN THE REINFORCED PLASTIC BOATBUILDING INDUSTRY}

Alysha Meyers, Steve Bertke, Avima Ruder. National Institute for Occupational Safety and Health, Cincinnati, Ohio, USA

\subsection{6/oemed-2014-102362.40}

Objectives To further evaluate the association of styrene, fibreglass, and wood dust exposure with non-malignant diseases, we extended follow-up through 2008 for 5203 workers exposed to styrene, fibreglass, and wood dust between 1959 and 1978 at two boat building plants.

Method We used a person-years analysis program, LTAS.NET to compute standardised mortality ratios (SMRs) using Washington State and U.S. rates, standardised rate ratios (SRRs), and 95\% confidence intervals. SMRs were stratified by exposure category (low or high) and duration of employment category ( $\leq 1$ year, $1+$ years).

Results Overall, 1206 nonmalignant deaths occurred (WA SMR 1.14, CI 1.08-1.21), with excess mortality for chronic obstructive pulmonary disease (COPD) overall $(\mathrm{n}=112$, WA SMR 1.61, CI 1.32-1.93), and among 2063 workers highly exposed to styrene and fibreglass ( $\mathrm{n}=39$, WA SMR 2.37, CI 1.693.25). Results were similar using U.S. mortality rates. Workers employed for less than one year had statistically significant increased mortality from several lifestyle-related outcomes (alcoholism, ischaemic heart disease, cirrhosis, accidental poisoning and homicide).

Conclusions The excess COPD mortality in this cohort is difficult to interpret. Recent reports associate styrene/fibreglass reinforced plastic manufacturing with another respiratory disease bronchiolitis obliterans. Based on a review of COPD death certificates, bronchiolitis obliterans does not appear to be a contributing factor for excess COPD mortality. The COPD excess in this study points to a need for an in-depth investigation of respiratory disease and occupational styrene exposure. Short term worker results are consistent with other occupational cohort studies.

\section{SHIFT WORK, LONG WORKING HOURS, AND PHYSICAL LABOUR IN RELATION TO MENSTRUAL FUNCTION: THE NURSES' HEALTH STUDY 3}

${ }^{1}$ Christina Lawson, ${ }^{1}$ Candice Johnson, ${ }^{2,3}$ Jorge Chavarro, ${ }^{3}$ Eileen Lividoti Hibert, 'Elizabeth Whelan, 'Carissa Rocheleau, ${ }^{1}$ Barbara Grajewski, ${ }^{2,3}$ Eva Schernhammer, 2,3 Janet Rich-Edwards. ${ }^{1}$ National Institute for Occupational Safety and Health, Cincinnati, OH, USA; ${ }^{2}$ Harvard School of Public Health and Harvard Medical School, Boston, MA, USA; ${ }^{3}$ Brigham and Women's Hospital, Boston, MA, USA

10.1136/oemed-2014-102362.41

Objectives We investigated associations between nursing occupational exposures and menstrual cycle regularity and cycle length.
Method Cross-sectional data were collected in 2010-2012 from 6309 nurses aged 21 to 45 from the Nurses' Health Study 3. We used multivariable regression modelling to analyse the associations between occupational exposures and prevalence of irregular cycles and long and short cycle lengths.

Results Cycle length was recorded as $<21$ days (1.5\%), 21-25 days (15.6\%), 26-31 days (69.7\%), and 32-50 days (13.2\%). In addition, $19 \%$ of participants reported irregular cycles. Working more than $41 \mathrm{~h} /$ week was associated with a 16\% [95\% confidence interval (CI): 4-29\%] higher prevalence of irregular cycles and a higher prevalence of very short $(<21$ day $)$ cycles [prevalence odds ratio (OR) 1.93, 95\% CI: 1.24-3.01]. Irregular menstrual cycles were more prevalent among women working nights only (32\% higher) or rotating nights $(27 \%$ higher), and their prevalence was associated with the number of night shifts per month ( $\mathrm{p}$ for trend $<0.0001$ ). Rotating night shift was also associated with long (32-50 day) cycles (OR 1.28, 95\% CI 1.031.61). In addition, heavy lifting was associated with a higher prevalence of irregular cycles (34\% higher), and the prevalence of cycles $<21$ days and 21-25 day cycles increased with increasing amount of heavy lifting at work ( $p$ for trend $<0.02$ for each endpoint).

Conclusions Night work, long working hours, and occupational physical labour might play a role in menstrual function disturbances.

\section{CLEANING TASKS AND RESPIRATORY, DERMATOLOGICAL AND MUSCULOSKELETAL SYMPTOMS AMONG CUSTODIANS USING TRADITIONAL AND GREEN CLEANERS}

${ }^{1}$ Jennifer Cavallari, ${ }^{1} J e n n i f e r$ Bruno Garza, 'Sara Wakai, ${ }^{2}$ Nancy Simcox, ${ }^{1}$ Paula Schenck, ${ }^{3}$ Loyola Welsh, ${ }^{4}$ John Meyer, ${ }^{1}$ Tim Morse, ${ }^{1}$ Martin Cherniack. ${ }^{1}$ University of Connecticut Health Center, Farmington, CT, USA; '2University of Washington, Seattle, WA, USA; ${ }^{3}$ Connecticut Employees Union Independent, Middletown, CT, USA; ${ }^{4}$ Mount Sinai Hospital, New York, NY, USA

\subsection{6/oemed-2014-102362.42}

Objectives As part of a larger study investigating the transition from traditional to green cleaners, we sought to investigate the relationships between cleaning tasks and respiratory, dermatological and musculoskeletal symptoms among a population of custodians.

Method State-employed custodians completed a questionnaire to assess cleaning tasks and health symptoms using standardised questions when available. Associations between health outcomes and cleaning tasks were investigated using logistic regression after controlling for age, gender, and smoking status. Each health outcome was modelled individually and trends with increasing exposures are reported.

Results Questionnaires were completed by 329 custodians from three universities and one university health centre. Participants were 59\% female, 53\% reported English as their primary language, and $18 \%$ were current smokers. Health symptoms within the last month included dermatitis (26\%), lower-respiratory complaints (30\%), upper-respiratory complaints (43\%), pain or discomfort in back (32\%) and pain or discomfort in neck, shoulders or arms (44\%). An increasing number of toilets cleaned was associated with increased odds of dermatitis ( $p$ for trend $=0.0005)$, lower-respiratory symptoms $(\mathrm{p}=0.007)$, and pain or discomfort in shoulders $(\mathrm{p}=0.04)$. Increasing daily hours spent floor stripping was associated with increased odds of dermatitis $(\mathrm{p}=0.02)$, lower- $(\mathrm{p}=0.01)$ and upper- $(\mathrm{p}=$ $0.01)$ respiratory symptoms as well as pain or discomfort in back 
$(\mathrm{p}=0.006)$. Increasing daily hours of vacuuming was associated with increased odds of lower- $(p=0.03)$ and upper- $(0.003)$ respiratory symptoms.

Conclusions Custodian dermatological, respiratory, and musculoskeletal symptoms are consistent with task related exposures and follow a dose-related pattern with increasing odds of symptoms related to increased exposure duration.

\section{APPROACHES TO DEVELOPING EXPOSURE ESTIMATES THAT REFLECT TEMPORAL TRENDS IN TOTAL PARTICULATE MATTER IN ALUMINIUM SMELTERS}

${ }^{1}$ Elizabeth Noth, ${ }^{1}$ Sa Liu, ${ }^{2}$ Mark Cullen, 'Ellen Eisen, 'S Katharine Hammond. 'University of California, Berkeley, Berkeley, CA, USA; ${ }^{2}$ Stanford University, Palo Alto, CA, USA

\subsection{6/oemed-2014-102362.43}

Objectives To evaluate different approaches for including time trends in quantitative exposures to total particulate matter (TPM) in an aluminium smelter for use in epidemiologic evaluation of incident heart disease, we compared the use of regression modelling to discrete modelling of changes in the workplace environment.

Method We used an industrial hygiene database containing results for sampling conducted over 30 years and information on workplace environment (e.g. personal protective equipment policy, ventilation modifications, changes to materials or work organisation). The effects of these changes were tested with an analysis of variance model using log-transformed TPM concentrations. We compared the outcome of this approach to the use of a regression model for TPM concentrations over time.

Results Time trends in 57 jobs in an aluminium smelter were evaluated by using 1123 TPM samples collected from 19842012. There was an overall decline in median TPM concentrations $\left(\mathrm{mg} / \mathrm{m}^{3}\right)$ at the smelter $(3.7 \%$ per year). The trend was not observed in the majority individual jobs. The decreasing trend was concentrated in $14 \%$ of the jobs. The majority of jobs (61\%) had no change over the time period, 19\% had no consistent pattern of change, and 5\% increased in TPM concentrations.

Conclusions Applying a global trend to worker exposures would result in misclassification error in epidemiologic evaluations. When possible, process changes should be used to define changes in worker exposures rather than using a facility- or industry-wide time trend. Future work will explore regression modelling as a way to explain any remaining time trends in TPM.

\section{PM2.5 AND HEART DISEASE IN A COHORT OF ALUMINIUM WORKERS: AN APPLICATION OF LONGITUDINAL TARGETED MAXIMUM LIKELIHOOD- BASED ESTIMATION (TMLE)}

'Daniel Brown, 'Maya Petersen, 'Mark van der Laan, 'Sadie Costello, 'Elizabeth Noth, ${ }^{1}$ Katherine Hammond, ${ }^{2}$ Mark Cullen, ${ }^{1}$ Ellen Eisen. ${ }^{1}$ UC Berkeley, Berkeley, CA, USA; ${ }^{2}$ Stanford University, Palo Alto, CA, USA

\subsection{6/oemed-2014-102362.44}

Objectives We estimated the effect of cumulative exposure to PM2.5 on the incidence of ischaemic heart disease (IHD) in aluminium workers followed for 15 years, adjusting for the healthy worker survivor effect. In previous analyses, higher cumulative exposure was found to be associated with lower mortality in this population.

Method We used longitudinal TMLE to estimate the cumulative risk of ischaemic heart disease in the cohort if constantly exposed above an exposure cut-off and compared it to the risk if constantly exposed below. We stratified all analyses by work process because exposures were an order of magnitude higher in smelters than fabrication facilities.

Results We selected cut-offs a priori at the median and 10th percentile exposure within each sub-cohort. Among the smelter workers, we estimated an increase in IHD risk of $2.1 \%(\mathrm{p}=$ 0.22) after 15 years, comparing the always exposed to never exposed cohort using the median cut-off of $1.77 \mathrm{mg} / \mathrm{m}^{3}$. The difference was $2.9 \%(\mathrm{p}=0.01)$ using the 10 th percentile cutoff of $0.10 \mathrm{mg} / \mathrm{m}^{3}$. For the fabrication workers, the differences were $0.1 \%(\mathrm{p}=0.47)$ using the median cut-off of $0.20 \mathrm{mg} / \mathrm{m}^{3}$ and $2.5 \%(\mathrm{p}<0.01)$ for the 10th percentile cut-off of $0.06 \mathrm{mg} / \mathrm{m}^{3}$. Results are presented as adjusted survival curves, describing the estimated cumulative risk for each cohort under each exposure regimen.

Conclusions The TMLE estimator allows us to observe an association between cumulative PM2.5 exposure and heart disease that was not visible using standard analytical techniques. This work represents the first application of longitudinal TMLE to the field of occupational epidemiology.

\section{PESTICIDE USE AND RELATIVE TELOMERE LENGTH IN THE AGRICULTURAL HEALTH STUDY}

${ }^{1}$ Gabriella Andreotti, ${ }^{2}$ Jane Hoppin, 'Sharon Savage, ${ }^{3}$ Lifang Hou, ${ }^{4}$ Andrea Baccarelli, ${ }^{4}$ Mirjam Hoxha, ${ }^{1}$ Stella Koutros, ${ }^{2}$ Dale Sandler, ${ }^{1}$ Michael Alavanja, ${ }^{1}$ Laura Beane Freeman. ${ }^{1} \mathrm{NCl}$, Rockville, MD, USA; ${ }^{2} \mathrm{NIEHS}$, Research Triagngle, NC, USA; ${ }^{3}$ Northwestern University, Chicago, IL, USA; ${ }^{4}$ University of Milan, Milan, Italy

\subsection{6/oemed-2014-102362.45}

Objectives Epidemiologic studies have linked pesticide use to various health outcomes, including cancer, but underlying mechanisms remain unclear. In a previous analysis from the Agricultural Health Study (AHS), a prospective cohort study of pesticide applicators in the US, use of certain pesticides was linked to shorter relative telomere length (RTL) measured in buccal cell DNA. In this analysis we examined the associations between occupational pesticide use and RTL measured in blood DNA.

Method We conducted an analysis of 80 pesticides and RTL in 568 cancer-free male participants aged 31-94 years in the AHS. We used self-reported pesticide use information collected at study enrollment (1993-1997) and two follow-up questionnaires administered approximately 5 years apart to construct exposure metrics, including intensity-weighted lifetime days (lifetime day$\mathrm{s}^{*}$ intensity score). Blood samples were collected in 2006-2008, and RTL was measured in DNA using qPCR. Multivariable linear regression was used to evaluate the associations between individual pesticide use and RTL, adjusting for age at blood draw and other pesticides associated with RTL.

Results Increasing tertiles of intensity-weighted days of alachlor were associated with longer RTL ( $\mathrm{p}$-trend $=0.01$ ). In contrast, increasing tertiles of intensity-weighted days of 2,4-D (p-trend $=$ $0.05)$, diazinon ( $\mathrm{p}$-trend $=0.01$ ) and aldrin $(\mathrm{p}$-trend $=0.01$ ) were associated with shorter RTL.

Conclusions We found two herbicides (alachlor, 2,4-D) and two insecticides (diazinon, aldrin) significantly associated with alterations in RTL. These pesticides have been linked to increased 